\title{
The Dynamics of the HD 12661 Extrasolar Planetary System
}

\author{
Adrián Rodríguez and Tabaré Gallardo \\ Departamento de Astronomía, Facultad de Ciencias, Iguá 4225, 11400 Montevideo, \\ Uruguay \\ arodriguez@fisica.edu.uy, gallardo@fisica.edu.uy
}

\begin{abstract}
The main goal of this work is to analyze the possible dynamical mechanisms that dominate the motion of the HD 12661 extrasolar planetary system. By an analytical approach using the expansion of the disturbing function given by Ellis \& Murray (2000) we solve the equation of motion working in a Hamiltonian formulation with the corresponding canonical variables and by means of appropriate canonical transformations. Comparing this results with a direct numerical integration we can conclude that the system is dominated by a pure secular evolution very well reproduced with a disturbing function including at least sixth order terms in the eccentricities. Because of the uncertainties in the orbital elements of the planets, we also contemplate the occurrence of mean-motion resonances in the system and analyze possible contribution from these resonant terms to the total motion.
\end{abstract}

Subject headings: canonical variables, celestial mechanics, extrasolar planets, secular evolution.

\section{Introduction}

There are some characteristics of the extrasolar planetary systems that make them different from the solar system, like the presence of massive planets close to the central stars (i.e, small values of semimajor axis) and with large values in the orbital eccentricities. Large eccentricities and small semimajor axis are the result of early migration processes where the planets were driven by tidal interaction with the disc where they were formed (Papaloizou 2003). Also, preliminary orbital determinations suggest the existence of resonant systems like Gliese 876 and HD 82943 with two planets locked in a 2:1 mean-motion resonance, 55 Cnc (3:1), 47 UMa (7:3)(Beaugé \& Michtchenko 2003, Hadjidemetriou 2002). These characteristics make classical perturbation theories originally developed for the solar system, like 
the Lagrange-Laplace secular theory, very limited to deal with the dynamics of extrasolar planets.

The system HD 12661 that we study here is composed by two massive planets around a central star with a relatively low semimajor axis ratio $\alpha \simeq 0.29$ (see Table 1 ). Some attempts have been done to explain the dynamical behavior of this system. In most of them, stability analysis are considered estimating the dynamical limits on the orbital parameters that provide quasi-periodic motions of the system (Goździewski 2003). The difficult to get the desired accuracy in the orbital fits in most of the extrasolar planets produces very important differences between similar works. This is also the case for HD 12661 where there are different results published according to the orbital elements taken. The system was supposed very affected by the 2:11 or 1:6 mean-motion resonance according to data published by Fischer et al. (2003) or new fits in the same year respectively (Goździewski and Maciejewski 2003, Ji et al. 2003). There are few analytical treatments applied to this particular case, unless the study by Lee \& Peale (2003) (see also Nagasawa et al. 2003 for a low order treatment in a cosmogonic framework) where they give an approach by means of the so called octopole secular theory. In that formulation using Jacobi coordinates the authors use a Hamiltonian function including up to third order terms in $\alpha$ and some fourth order terms in eccentricities. They find a qualitatively good description for the system but severe discrepancies between the frequencies predicted by the theory and the ones computed from the numerical integration of the full equation of motion. In this paper we present a semi-analytical theory that explains the dynamical evolution of the system.

The present paper is organized as follow: In section 2 we present the actual data of the system, perform a numerical integration using the orbital fits by Debra Fischer (2004, private communication) and discuss the possible effects of the main mean motion resonances near the system. The canonical variables necessary to work in an analytical framework are presented in section 3. In section 4 we compute the value of the disturbing function to several orders in eccentricities through the Ellis \& Murray (2000) expansion and investigate the contributions of possible mean-motion resonances. In section 5 we present and resolve our secular model and compare with numerical integrations. Discussion and finals remarks are devoted to section 6 . 


\section{The HD 12661 Extrasolar System}

Some authors considered the possibility of this system being strongly affected by a mean motion resonance. In particular Lee \& Peale (2003) suggested that discrepancies between their octopole model and the direct numerical integration could be due to the 2:11 resonance. Different orbital determinations according to different data sets and methods (Lee \& Peale 2003, Goździewski \& Maciejewski 2003) lead to substantial differences in the semimajor axis of the exterior planet. On the contrary, the semimajor axis of the internal planet seems to be well determined according to the successive orbital determinations. For illustration purposes at Figure 1 we show the approximate position of the most important exterior mean motion resonances deduced from the simple relation $a=a_{1}(p /(p+q))^{2 / 3}$ together with the most probable location of the exterior planet according to Table 1 . The parameter $p$ is the degree of the resonance and is taken positive for interior resonances and negative for exterior ones. The parameter $q$, always positive, is the order. The width of each resonance is not indicated, we refer to Goździewski \& Maciejewski (2003) for a numerical estimation of the width of the most important resonances. The exterior planet is very near the 2:13 and 3:19 resonances, but both are very high order resonances. In an expansion of the disturbing function for quasi planar orbits the most important resonant terms are of order $q$ in the eccentricities. Then that terms are of order 11 and 16 in eccentricity respectively and for small and medium eccentricity values as in this particular system in principle we cannot expect those terms to became relevant for the dynamical evolution of the system. But, in spite of not being important for the approximate description of the system, some of those slight effects could be detected with numerical methods like MEGNO (Cincotta \& Simó 2000) as was clearly shown by Goździewski \& Maciejewski (2003). We will discuss more profoundly this point at section 4 .

We perform a numerical integration of the HD 12661 extrasolar planetary system supposed coplanar, with $\sin i=1$ and with a central star mass of $1.07 M_{\odot}$. The main goal is to compare the results with an analytical model to find the dynamical mechanisms that dominate the system. The orbital elements of HD 12661 had recently changed from their last values (Fischer et al. 2003). The astrocentric osculating orbital elements used here to make the numerical integrations are presented at Table 2 and were deduced from Table 1 assuming the radial velocity fit for the planet $\mathrm{c}$ better emulate an orbit referred at the baricenter of the star and planet b as suggested by Lissauer \& Rivera (2001). The numerical integrator used was MERCURY (Chambers 1999). 


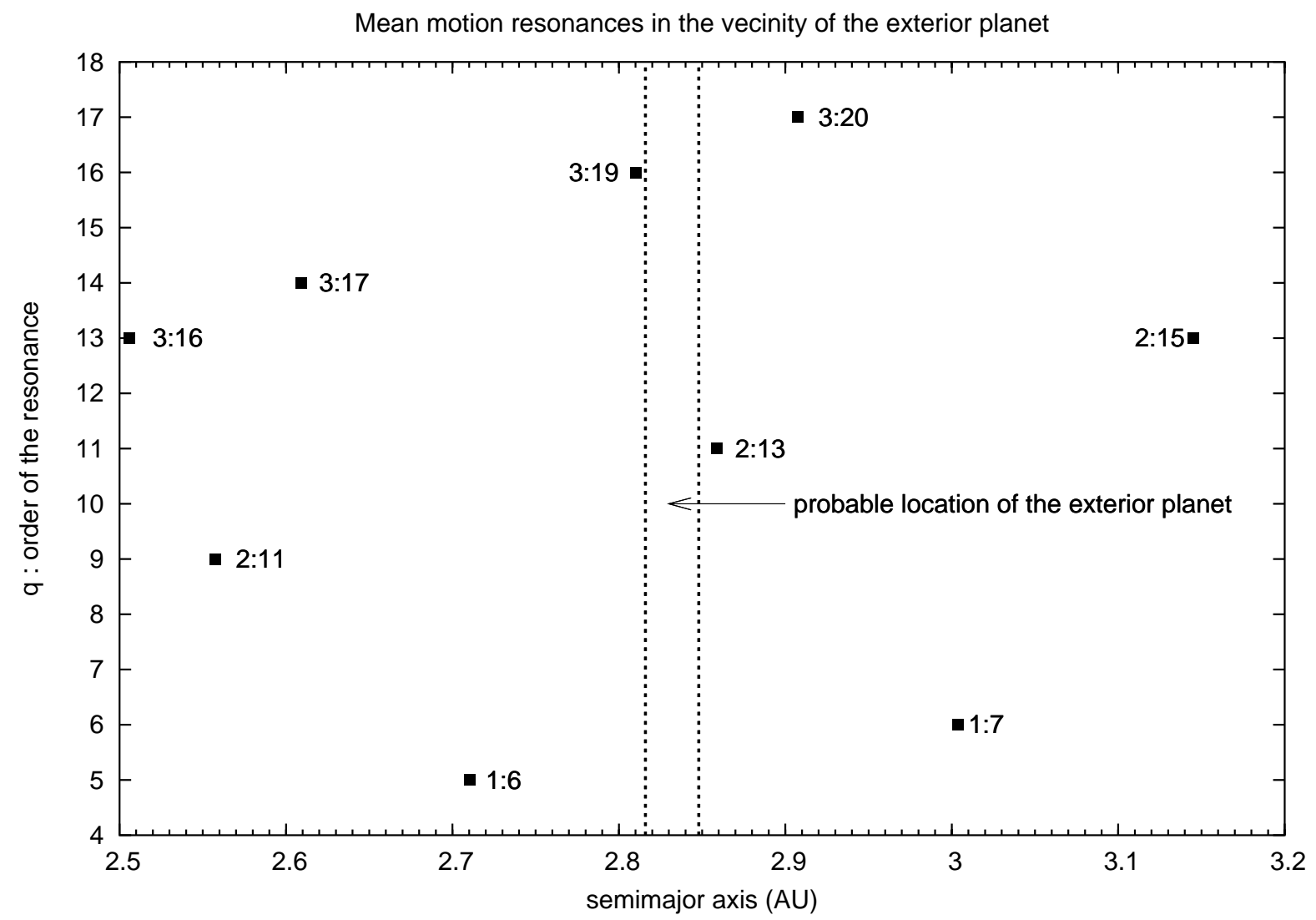

Fig. 1. - Approximate localization of the exterior mean motion resonances $|p+q|:|p|$ near the exterior planet. Higher order resonances should have less dynamical signatures. It should be kept in mind that each resonance covers some range $\Delta a$ that is not indicated here. The semimajor axis of the exterior planet was taken from Table 1 which is approximately equal to its mean semimajor axis.

\begin{tabular}{cccccccc}
\hline \hline & & & & & & & \\
Planet & $P($ days $)$ & $K(\mathrm{~m} / \mathrm{s})$ & $e$ & $\omega($ deg $)$ & $T p(M J D)$ & $m\left(M_{\text {Jup }}\right)$ & $a(A U)$ \\
\hline & & & & & & & \\
HD 12661b & 262.37 & 75.02 & 0.34 & 296.6 & 9952.267 & 2.33 & 0.821 \\
Error $(+/-)$ & 0.32 & 0.85 & 0.02 & 2.6 & 3.6 & 0.02 & 0.001 \\
HD 12661c & 1681.7 & 29.9 & 0.06 & 102.8 & 10314.221 & 1.83 & 2.834 \\
Error $(+/-)$ & 14.2 & 0.93 & 0.02 & 77.9 & 641.7 & 0.06 & 0.016 \\
\hline \hline
\end{tabular}

Table 1: Parameters $P, K, e, \omega$ and $T_{p}$ are from two-Kepler fits by Debra Fischer (2004, private communication). The errors were estimated by a Monte Carlo method. Parameters 
$m$ and $a$ were deduced following formulae 2,4,6 and 8 from Lee \& Peale (2003) assuming $\sin i=1$ and a stellar mass of $1.07 M_{\odot}$.

\begin{tabular}{ccccc}
\hline \hline & & & & \\
Planet & $a(A U)$ & $e$ & $\omega($ deg $)$ & $M($ deg $)$ \\
\hline & & & & \\
HD 12661b & 0.821 & 0.34 & 296.6 & 136.7 \\
Error $(+/-)$ & 0.001 & 0.02 & 2.6 & 4.9 \\
HD 12661c & 2.855 & 0.066 & 102.1 & 0.7 \\
Error $(+/-)$ & 0.016 & 0.02 & 77.9 & 137 \\
\hline \hline
\end{tabular}

Table 2: Astrocentric osculating orbital elements deduced from Table 1 for the epoch JD 2450314.22

The Figures 2 and 3 show the time evolution of the astrocentric semi-major axes and eccentricities for the two planets. We can see the periodic evolution in eccentricities showing an almost perfect coupling between them, i.e both elements oscillates with the same period. This is a simple consequence of the conservation of the total angular momentum of the system, which is approximately equal to the angular momentum of the planets because the angular momentum of the star with respect to the barycenter is negligible. Then, the summation of the angular momentum of the planets is almost conserved and this explain the coupling between the eccentricities. A rigorous explanation can be found in Ferraz-Mello et al. (2004). On the other hand, the semi-major axis $a_{2}$ shows very small oscillations with frequency equal to the synodic frequency of the planetary system. These short period oscillations in the osculating astrocentric $a_{2}$ almost disappear when working in a reference system with center at the baricenter of the star and the first planet. It seems that the dynamics is mainly driven by a typical secular behavior. However, this is only a suggestion because we must demonstrate it by means of the analysis of the disturbing function for this particular system, isolating the secular terms in a particular development, resolving the equation of motion and then comparing the results with the ones obtained by the direct numerical integration.

From Figure 3 we see that the eccentricities oscillate in the ranges $0.14<e_{1}<0.34$ and $0.04<e_{2}<0.27$ reaching periodically high eccentric orbits. By means of a spectral analysis (Gallardo \& Ferraz-Mello 1997) we found a value of $T_{e}=26150 \mathrm{yrs}$ for the period in the time evolution of the eccentricities. This parameter will serve as reference for the results obtained here and those calculated after in an analytical context. 


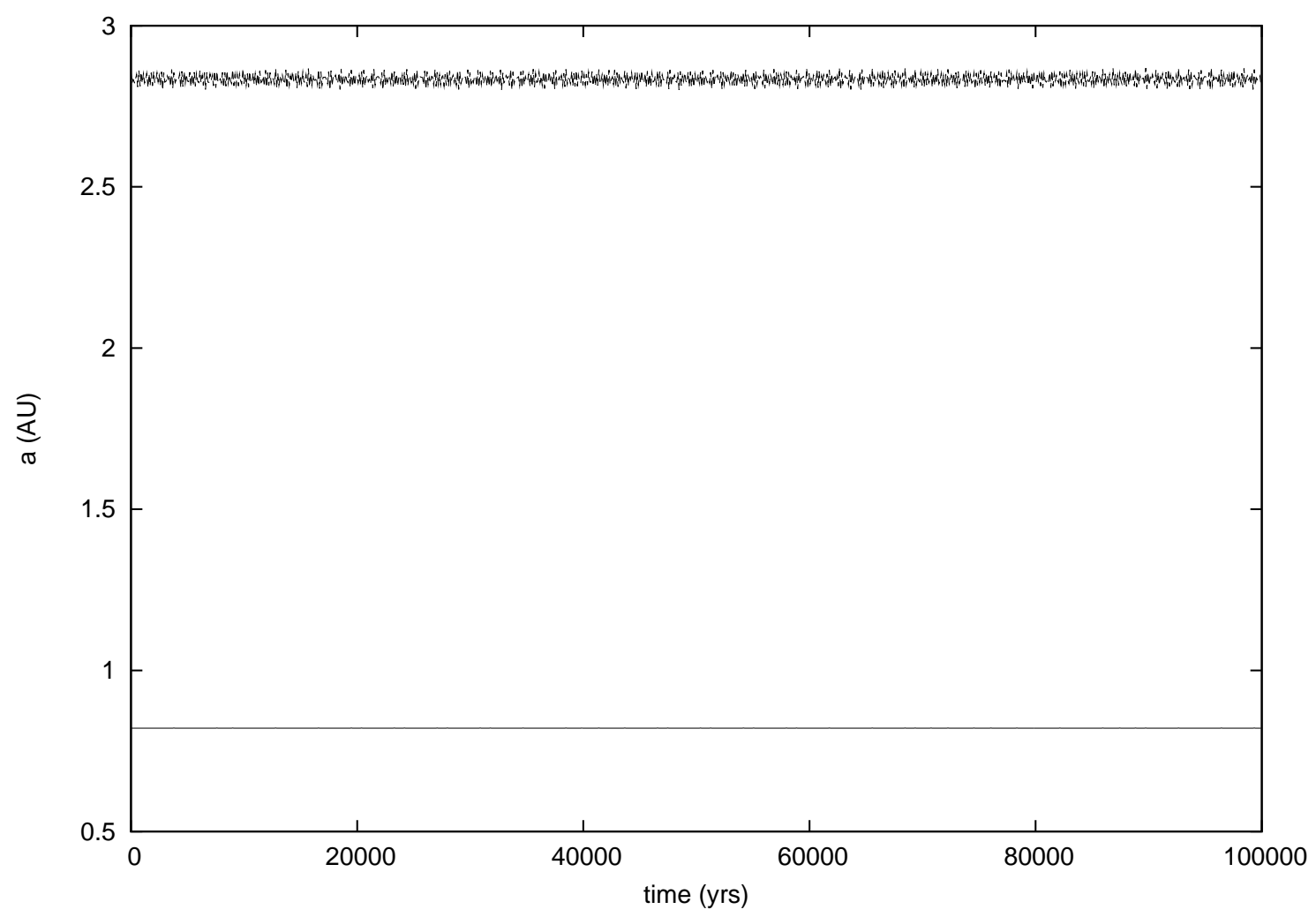

Fig. 2. - Time evolution for the astrocentric semi-major axes. Initial orbital elements taken from Table 2. Elapsed time from the epoch.

We refer the reader to Figure 7 were we show the evolution in the plane $\left(e_{1} \cos \Delta \varpi\right.$, $\left.e_{1} \sin \Delta \varpi\right)$, where $\Delta \varpi \equiv \varpi_{1}-\varpi_{2}$ is the difference of longitude of pericenter of the planets and $e_{1}$ is the eccentricity of planet $b$. These variables represent a modification of the classical $(k, h)$ ones that appear in the usual literature (Murray \& Dermott 1999). The vector going from the origin to any point of the picture gives the value of the eccentricity at this point. We see in the figure that the angle $\Delta \varpi$ exhibit a libration around $\pi$, meaning that the pericenters are averagely anti-aligned. In fact, this was the first discovered extrasolar system showing that behavior (Lee \& Peale 2003). We mention again that this behavior as the previous found results will be contrasted in the next sections with the corresponding analytical model to deal the dynamics of the system. Moreover, we will try to answer if it is the dynamical evolution explained by a pure secular effect or on the contrary it is dominated by another mechanism like a mean-motion resonance. Now let us work with the mathematical formulation of the problem. 


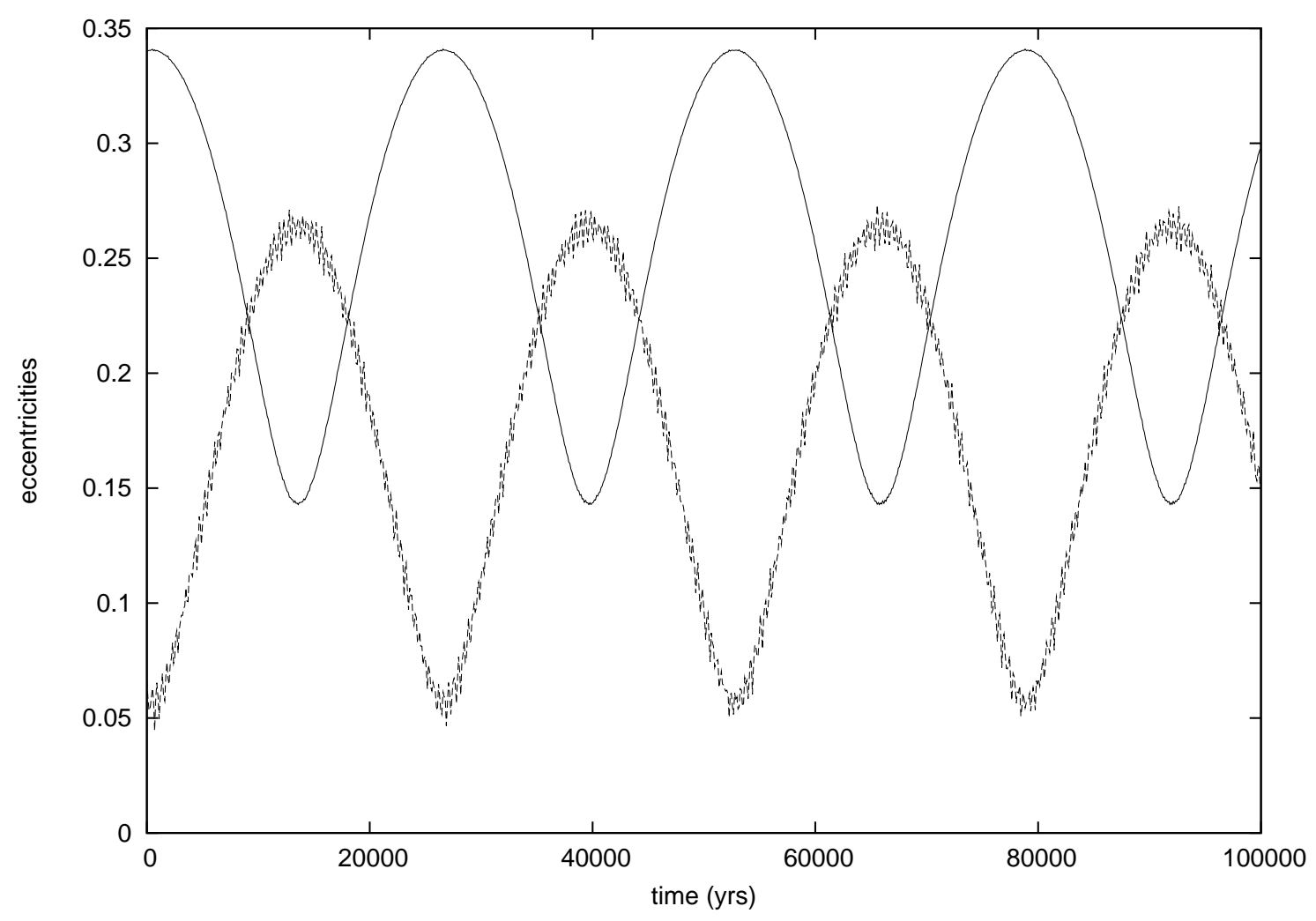

Fig. 3.- Time evolution for the eccentricities. Initial orbital elements taken from Table 2. The coupling between eccentricities is a natural secular behavior for systems with two planets. Elapsed time from the epoch.

\section{Hamiltonian Equations for the Two-Planet Problem}

In this section we will obtain the differential equations describing the dynamical evolution of the system. We can use the Langrange's planetary equations but we prefer to follow a Hamiltonian formulation as is explained in Morbidelli (2002) or Ferraz-Mello et al. (2004). Although it seems to be an easy problem, most papers referred to the three-body problem in celestial mechanics deal with the so-called restricted three-body problem, where only two bodies have finite masses. Here, we begin with a presentation of the general three-body problem and then define the adequate canonical variables necessary to work in the Hamiltonian formalism.

Let us consider the system composed by three objects (a central star plus two planets) with masses $m_{i}(i=0,1,2)$ subject to their mutual gravitational attraction. Let $O$ the barycenter of the system and $\mathbf{R}_{i}, \mathbf{P}_{i}=m_{i} \dot{\mathbf{R}}_{i}$ the coordinates and linear momenta in the 
barycentric reference frame, i.e respect to $O$. These variables are canonical then we can write the Hamiltonian of the system in the form:

$$
H=\frac{1}{2} \sum_{i=0}^{2} \frac{P_{i}^{2}}{m_{i}}-G \sum_{i=0}^{2} \sum_{j=i+1}^{2} \frac{m_{i} m_{j}}{\Delta_{i j}}
$$

where $\Delta_{i j}=\left|\mathbf{R}_{i}-\mathbf{R}_{j}\right|$ is the relative distance between the bodies and $G$ the constant of gravitation. It is not other thing than the sum of the kinetic and potential energy of the system, $H=T+U$. This system has nine degrees of freedom (i.e, $3(N+1)$ in the general $N$-planet plus star problem) and using the adequate sets of canonical variables it can be reduced to six taking into account the conservation laws concerning the inertial motion of the barycenter.

\subsection{Canonical Astrocentric Variables}

We will follow here the Poincaré's reduction of degrees of freedom by means of a canonical transformation. It consist in taking the coordinates and momenta as

$$
\mathbf{r}_{i}=\mathbf{R}_{i}-\mathbf{R}_{0} \quad \mathbf{p}_{i}=\mathbf{P}_{i} \quad i=1,2
$$

The choice of the variables is such as the coordinates are the astrocentric position vectors and the linear momenta are the same that those in the barycentric formulation. To complete the transformation we need to define

$$
\mathbf{r}_{0}=\mathbf{R}_{0} \quad \mathbf{p}_{0}=\sum_{i=0}^{2} \mathbf{P}_{i}
$$

Now $\left(\mathbf{r}_{i}, \mathbf{p}_{i}\right),(i=0,1,2)$ are canonical and we can express the above Hamiltonian in the new variables. To do this, we split the whole Hamiltonian in the sum of the kinetic and potential energy and express each one in the new variables, then we add both parts and obtain the final expression. Doing that we see that $\mathbf{r}_{0}$ do not appear in the Hamiltonian, so it is an ignorable variable. Therefore $\mathbf{p}_{0}$ is a constant that we can set equal zero by construction (the linear momentum of the system). In this way, we reduce the degrees of freedom from nine to six (see details in Ferraz-Mello et al. 2004, Laskar \& Robutel 1995 and Morbidelli 2002):

$$
H=H_{0}+H_{1}
$$




$$
\begin{aligned}
& H_{0}=T_{0}+U_{0}=\sum_{i=1}^{2}\left(\frac{p_{i}^{2}}{2 \beta_{i}}-\frac{\mu_{i} \beta_{i}}{r_{i}}\right) \\
& H_{1}=T_{1}+U_{1}=\sum_{i=1}^{2} \sum_{j=i+1}^{2}\left(-\frac{G m_{i} m_{j}}{\Delta_{i j}}+\frac{\mathbf{p}_{i} \cdot \mathbf{p}_{j}}{m_{0}}\right)
\end{aligned}
$$

where

$$
\mu_{i}=G\left(m_{0}+m_{i}\right) \quad \beta_{i}=\frac{m_{0} m_{i}}{m_{0}+m_{i}}
$$

and in analogy to Laskar \& Robutel (1995) we will call canonical astrocentric variables to the $\left(\mathbf{r}_{i}, \mathbf{p}_{i}\right),(i=1,2)$. We can see that $H_{0}$ is of the order of the planetary masses $m_{i}$ while $H_{1}$ is of order two with respect to this masses. Then $H$ is composed of the unperturbed contribution $H_{0}$ and the disturbing part $H_{1}$ (also called Disturbing Function, arising from the potential energy of the interaction between the planets), where the direct and indirect terms are identified. In contrast with the classical description of the disturbing function where the indirect part depend on the positions of the planets, here it depends on the velocities through the p's.

We also see that in the expression $5, H_{0}$ is composed by two terms of the form

$$
F_{i}=\left(\frac{p_{i}^{2}}{2 \beta_{i}}-\frac{\mu_{i} \beta_{i}}{r_{i}}\right)=\left(\frac{p_{i}^{2}}{2 \beta_{i}}-G \frac{m_{0} m_{i}}{r_{i}}\right)
$$

Each $F_{i}$ is the Hamiltonian of a two-body problem in which the mass $m_{i}$ is moving around the mass $m_{0}$. One of the Hamilton equations generated by $H_{0}$ is

$$
\dot{\mathbf{r}_{i}}=\frac{\mathbf{p}_{i}}{\beta_{i}}
$$

Then in analogy to the two body problem we can define orbital elements from $(\mathbf{r}, \mathbf{p})$ in spite of being referred to different origins ( $\mathbf{r}$ belonging from an astrocentric reference frame and $\mathbf{p}$ from a barycentric reference frame). The trouble with this is that the actual value of $\dot{\mathbf{r}}_{i}$ will have a contribution from $H_{1}$ in the equation of motion:

$$
\dot{\mathbf{r}}_{i}=\frac{\partial H_{0}}{\partial p_{i}}+\frac{\partial H_{1}}{\partial p_{i}}
$$

and in consequence the actual astrocentric velocity $\dot{\mathbf{r}}$ will not follow the direction given by $\mathbf{p}$, and that means we are defining ellipses which are not tangent to the real trajectory. These ellipses they no more represent "osculating" ellipses. We recall that osculating means "kissing" or tangent ellipses. This particularity, as suggested by Laskar \& Robutel (1995), is probably the reason that explain their few usage. We will call canonical orbital elements 
to the orbital elements defined from the astrocentric canonical variables to distinguish from the usual osculating orbital elements.

An alternative to the canonical astrocentric variables from Poincare are the Jacobi coordinates used by Lee \& Peale (2003) in their octopole model. For hierarchical planetary systems the orbital elements defined from Jacobi coordinates have the advantage of exhibiting lower amplitude short period perturbations.

\subsection{The Canonical Orbital Elements}

Once we know the $(\mathbf{r}, \mathbf{p})$ it is possible to reconstruct the orbit of each planet considering the unperturbed part of the whole Hamiltonian. If the problem is expressed as the perturbation of an integrable Hamiltonian $H_{0}$, it is natural to use action-angle variables as canonical coordinates to resolve the corresponding Hamilton-Jacobi equations. In that context it is classical to define the Delaunay variables as action-angle variables and construct the corresponding orbital elements from them. However here we have $H_{0}$ defined as a function of $\mathbf{p}_{i}$ which depends of the reduced mass $\beta_{i}$. So the corresponding Delaunay variables will be affected by this modification, and for the planar case we obtain:

$$
\begin{aligned}
\lambda_{i} & \Lambda_{i} & =\beta_{i} \sqrt{\mu_{i} a_{i}} \\
-\varpi_{i} & \Gamma_{i} & =-\Lambda_{i}\left(1-\sqrt{1-e_{i}^{2}}\right)
\end{aligned}
$$

$(i=1,2)$, where $\mu_{i}, \beta_{i}$ are defined as before in (7) and $a$,e are the canonical orbital elements introduced by the definitions (for each planet)

$$
\begin{array}{r}
a=\frac{\mu r}{2 \mu-r w^{2}} \\
e=\sqrt{\left(1-\frac{r}{a}\right)^{2}+\frac{(\mathbf{r} \cdot \mathbf{w})^{2}}{\mu a}}
\end{array}
$$

where $\mathbf{w}$ is not the astrocentric instantaneous velocity but $\mathbf{w}=\mathbf{p} / \beta$. The angles $\lambda$ and $\varpi$ for each planet are obtained with the usual equations for the planar problem. We refer to Ferraz-Mello et al. (2004) for more details.

A very important simplification occurs when considering the secular part, $\bar{H}_{S e c}$, of $H$. 
As can be shown (Ferraz-Mello et al. 2004);

$$
\frac{\partial \bar{H}_{S e c}}{\partial p_{i}}=0
$$

there is no contribution to $\dot{\mathbf{r}}$ from $\bar{H}_{S e c}$ and then from the equation of motion

$$
\dot{\mathbf{r}_{i}}=\frac{\mathbf{p}_{i}}{\beta_{i}}
$$

which implies $\mathbf{w}_{i}=\dot{\mathbf{r}}_{i}$ so there is no difference between the canonical orbital elements and usual astrocentric Keplerian elements. We remark: when studying the secular evolution of the system, the usual Keplerian orbital elements are equal to the canonical orbital elements. In both cases we must understand they are mean elements because we are dealing with the secular terms of the disturbing function.

\section{The Disturbing Function for Moderate Eccentricities}

In order to obtain a good analytical description of the system it is necessary to work with an adequate expression for the disturbing function which is part of the Hamiltonian of the system. As numerical integrations show, the planetary eccentricities of this system can reach values as high as 0.34. A disturbing function truncated to second order in the eccentricities most probably will fail in the attempt to describe correctly the dynamical evolution of this system. Due to the same reason the Lagrange-Laplace secular planetary theory obtained by a second order perturbing function with simplified Lagrange's planetary equations (Murray \& Dermott 1999, Morbidelli 2002) neither can be applied. In this section we will explore how many terms we need to consider for the disturbing function. This will be done looking at the contribution given by terms of successive higher order.

The disturbing function can be expanded in several ways. The reader can refer to Ellis \& Murray (2000) for a review on classical expansions and to Beaugé \& Michtchenko (2003) for an alternative viewpoint for the expansion which can be applied even when the orbits intersect. In this paper we will use the expansion given by Ellis \& Murray (2000). An alternative expansion can be found in Laskar \& Robutel (1995).

Assuming planar orbits the disturbing function for both planets will depend on $\left(\alpha, e_{1}\right.$, $\left.e_{2}, \lambda_{1}, \lambda_{2}, \varpi_{1}, \varpi_{2}\right)$ and also on the masses of the three bodies. It can be shown via averaging methods that to first order in the planetary masses the Hamiltonian which describe the long term evolution of the system (i.e. the secular evolution of the system) can be obtained from the secular part of the disturbing function, that means, the terms not depending on the quick varying mean longitudes. It is also possible to show the secular part will be a function 
of $\left(\alpha, e_{1}, e_{2}, \Delta \varpi\right)$ (Brouwer \& Clemence 1961).

To determine the order of the secular part of the disturbing function necessary for a correct description of the long term evolution of the system we have plotted at Figures 4 and 5 the secular disturbing function as a function of $e_{1}$ for two values of $e_{2}$ (0.1 and 0.4 ) and assuming $\Delta \varpi=\pi$. The value of $\alpha$ was taken equal to the nominal one from Table 1 . The different curves represent the expansion of the secular part of the disturbing function truncated at different orders. For the case in which $e_{2}$ is small the curves corresponding to truncation to 4, 6 and 8 order are coincident, then we can expect an expansion truncated at fourth order will give enough precision. But, when $e_{2}$ is greater a sixth order or eight order expansion will be required for a correct analytical description.

We also plotted the contribution from the terms corresponding to the resonance 1:6 (evaluated at $\lambda_{1}=0$ and $\lambda_{2}=\pi$ ) which is distinguishable for the case $e_{2}=0.4$ at the higher values of $e_{1}$ (Fig. 5). It is important to stress that the system never gets so high values for both eccentricities simultaneously. This is a very small contribution which a precise numerical analysis like MEGNO could detect. In a similar way we also computed the contribution of the terms corresponding to the 2:13 resonance. We found that the contribution of these terms is completely negligible and of order $10^{-7}$ in the same relative units of the figures.

From this analysis we can conclude that it is necessary at least a sixth order expansion for the secular disturbing function for describing the secular evolution of the system. We also can conclude that for the most probable values of $\alpha$ and for the range of values exhibited by $e_{1}$ and $e_{2}$ we cannot expect evident dynamical effects associated with mean motion resonances. As shown in section 2, according to the observational data, there are no low order resonances near the system and then there is no reason for considering resonant terms. Anyway if an eight order secular disturbing function do not reproduce the results obtained by the numerical integration of the full equations of motion we will have to consider those resonant terms.

\section{Secular Evolution of HD 12661}

Once we test the Ellis \& Murray development of the disturbing function, our task now is to use this expansion to make an analytical exploration of the dynamics of the HD 12661 extrasolar planetary system. We suppose that the system is not locked in a mean-motion 


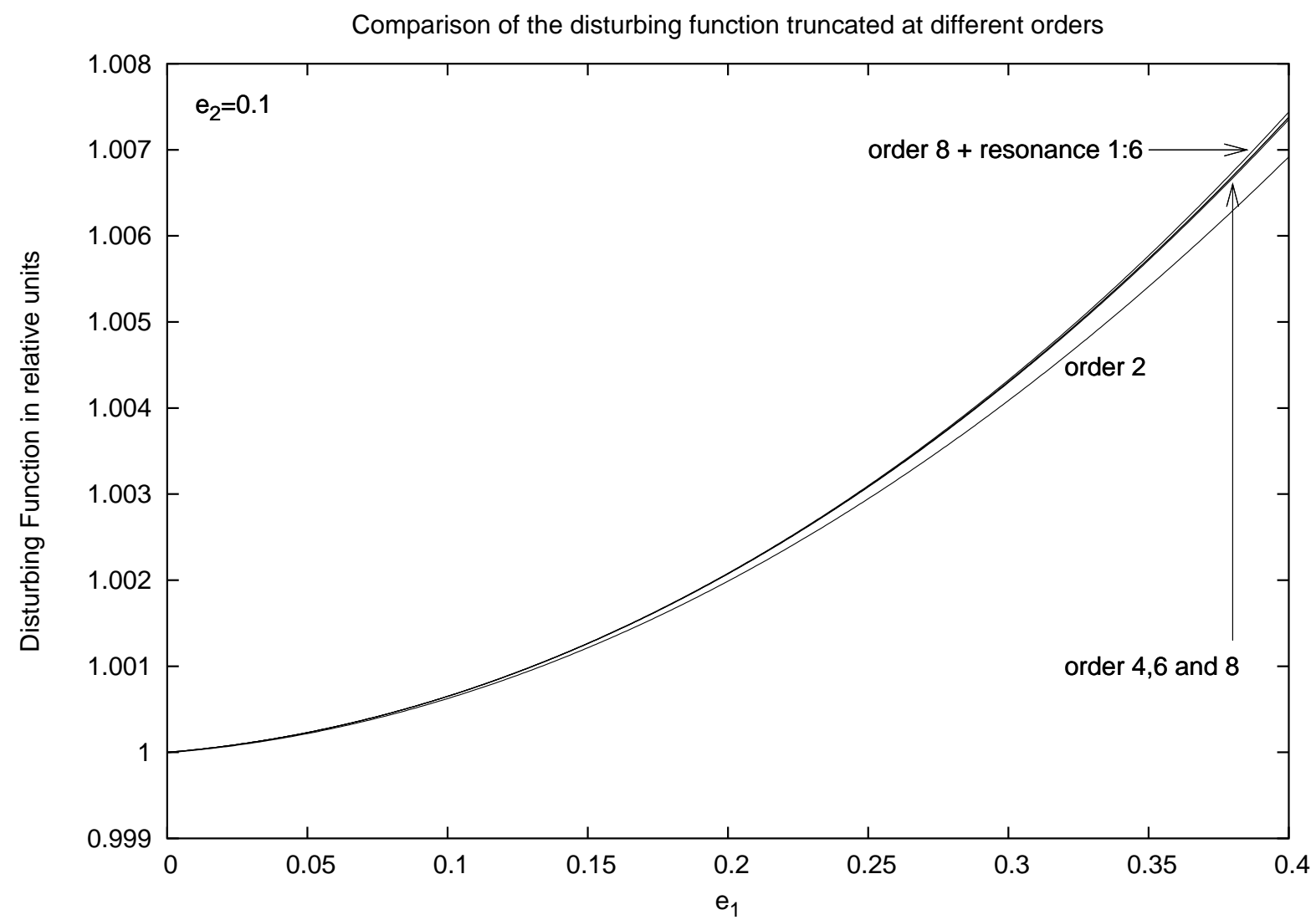

Fig. 4. - The secular part of the disturbing function in relative units truncated at different orders in the eccentricities and evaluated for $e_{2}=0.1$ and $\Delta \varpi=\pi$. The contribution from terms of orders sixth and eight are negligible. The resonant terms were evaluated at $\lambda_{1}=0$ and $\lambda_{2}=\pi$.

resonance, so the dynamics will be dominated by the secular evolution of the system. Then we will compare the results obtained by this model with the numerical integration of the full equations of motion. We will begin with the definition of the adequate canonical variables to deal with the secular evolution in order to integrate then the corresponding Hamilton equations of motions.

\subsection{Canonical Variables for the Secular Dynamics}

We want to explore the dynamical behavior of the system studying the time evolution of the orbital elements of the planets. To do this, we first need to define and resolve the Hamilton equations of motion. We will work based on the canonical variables defined in 


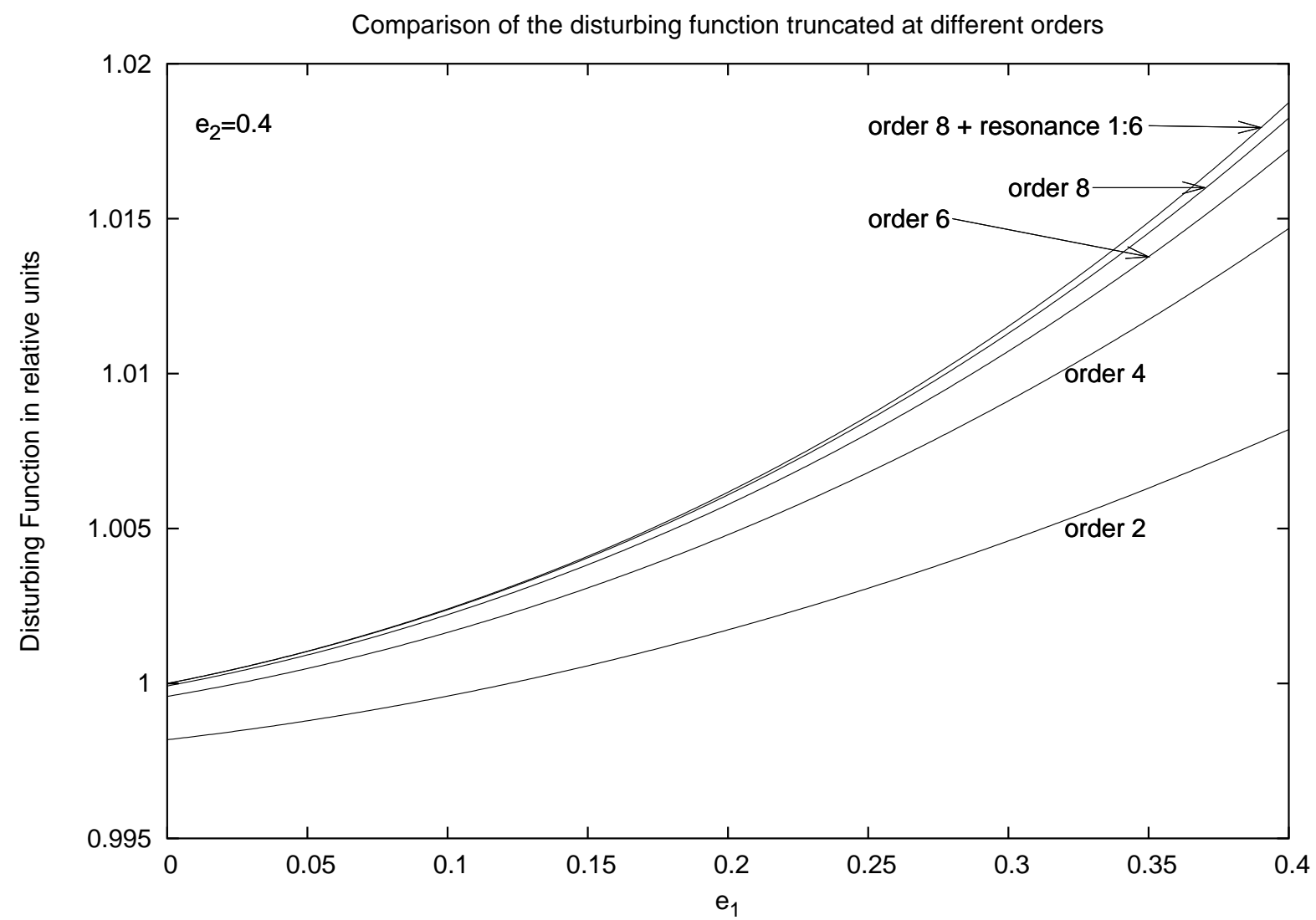

Fig. 5. - The secular part of the disturbing function in relative units truncated at different orders in the eccentricities and evaluated for $e_{2}=0.4$ and $\Delta \varpi=\pi$. The contribution from terms of orders sixth and eight are appreciable. The resonant terms were evaluated at $\lambda_{1}=0$ and $\lambda_{2}=\pi$

section 3 with some modifications that become adapted to deal with the secular evolution (Michtchenko \& Ferraz-Mello 2001). Moreover, working in a secular context we need to express the development of the disturbing function in terms of the mean variables. These are:

$$
\begin{aligned}
\lambda_{i} & \Lambda_{i} \\
\Delta \varpi=\varpi_{1}-\varpi_{2} & K_{1}=\Gamma_{1} \\
\phi=-\varpi_{2} & K_{2}=\Gamma_{1}+\Gamma_{2}
\end{aligned}
$$

where $i=(1,2)$ and $\Lambda_{i}, \Gamma_{i}$ defined as before at (11) and (12). Since we have a secular Hamiltonian, it does not depend on the mean longitudes $\lambda_{i}$, so the corresponding momenta 
$\Lambda_{i}$ are two constant of motion that we call $\Lambda_{i}^{*}$. This shows from (11) that the semi-major axes keep constant during a purely secular evolution. Moreover, the averaged planar Hamiltonian has a $\phi$ dependence only trough $\Delta \varpi$ (see Brouwer \& Clemence 1961), so $\phi$ is a cyclic coordinate and therefore their momenta $K_{2}$ is also a constant of motion related to the angular momentum of the system that we call $K_{2}^{*}$. With this simplifications, we will have a secular Hamiltonian:

$$
\bar{H}_{S e c}=-\sum_{i=1}^{2} \frac{\mu_{i}^{2} \beta_{i}^{3}}{2\left(\Lambda_{i}^{*}\right)^{2}}-\mathcal{R}_{S e c}\left(\Delta \varpi, K_{1}, K_{2}^{*}, \Lambda_{1}^{*}, \Lambda_{2}^{*}\right)
$$

where $\mathcal{R}_{\text {Sec }}$ is the secular part of the disturbing function expressed in terms of the canonical variables. This means that the averaged planar three-body problem it has one degree of freedom and therefore it is integrable. Therefore, the secular solution of the system it has an important consequence: the solutions for $\Delta \varpi$ and $K_{1}$ are periodic functions in the time with the same period. So, it means that the secular evolution is well described by a single frequency which its signature must be evident in the time evolution of the eccentricities and perihelion longitudes of both planets. The corresponding equations of motion for the pair $\left(\Delta \varpi, K_{1}\right)$ are:

$$
\Delta \dot{\varpi}=\frac{\partial \bar{H}_{S e c}}{\partial K_{1}} \quad \dot{K}_{1}=-\frac{\partial \bar{H}_{S e c}}{\partial \varpi}
$$

The two equations (17) for $\lambda_{i}$ are trivial and from them we obtain the two constants $\Lambda_{i}^{*}$ knowing the values for the initial semi-major axis. The last equation (19) is also trivial and we get the value of $K_{2}^{*}$ setting $\bar{H}_{S e c}\left(\Delta \varpi, K_{1}, K_{2}^{*}, \Lambda_{1}^{*}, \Lambda_{2}^{*}\right)=H^{*}$ for a given $H^{*}$ that can be calculated from the initial conditions for the system. The initial conditions for the secular system (initial mean orbital elements) are obtained from a short time span numerical integration of the full equations of motion. So, resolving the equations (21) we obtain the solutions for the canonical variables $\Delta \varpi(t)$ and $K_{1}(t)$. After that, we can use the inverse transformation to obtain the solution in terms of the mean orbital elements to describe the temporal secular evolution of the individual orbital eccentricities and relative longitude of pericenter of the planets $e_{1}(t), e_{2}(t)$ and $\Delta \varpi(t)$. As we noted in section 3 , the mean orbital elements are coincident with the canonical orbital elements when the system is driven only by secular terms. We refer also to Michtchenko \& Malhotra (2004), a semi-analytical work where they study the secular dynamics of a system compound by two massive planets trying with a similar secular model presented here. However, the dynamical analysis it is done without any particular development of the disturbing function, i.e the the study is valid for any value of the eccentricities. 


\subsection{Solutions for Successive Higher Orders}

We will numerically solve the secular equation of motion (21) using expansions of successive higher orders in the disturbing function $\mathcal{R}_{S e c}$ that appears in the secular Hamiltonian (20). We perform four different development in $\bar{H}_{S e c}$, using the disturbing function truncated to orders two, four, six and eight.

For each expansion we find the time evolution of the canonical variables $\left(\Delta \varpi, K_{1}\right)$ and the (mean) eccentricities of the planets constructed from them. Both $\Delta \varpi$ and $K_{1}$ are periodic functions with the same frequency and in fact this is the frequency present in the one degree of freedom secular problem. We compute the value of the oscillation period of the eccentricities $T_{e}$ and compare with the values obtained in the numerical integration of the full equations at section 2 . To compute $T_{e}$ we have used the same algorithm that in section 2. The results are presented at Table 3 . We remark that $\Delta \varpi$ is a periodic function with the same period that the eccentricities.

\begin{tabular}{c|c|c}
\hline \hline Order & $T_{e}^{\text {mod }}(\mathrm{yrs})$ & $T_{e}^{\text {mod }}-T_{e}^{\text {num }}(\mathrm{yrs})$ \\
\hline 2 & 30970 & $4820(18.4 \%)$ \\
4 & 26760 & $610(2.3 \%)$ \\
6 & 26380 & $230(0.9 \%)$ \\
8 & 26350 & $200(0.8 \%)$ \\
\hline
\end{tabular}

Table 3. Oscillation period of the eccentricities from the analytical models compared with the one deduced from the numerical integration of the full equations of motion.

We find that to order two the secular model fails in reproducing the oscillation period and amplitudes. In fact, a secular evolution to order two it is equivalent to the LaplaceLagrange secular theory, specially adapted to systems with very low values in the eccentricities. With the successive development to higher orders, the differences begin to decrease until the convergence is reached with a sixth order expansion. Therefore we can say that for this particular case, at least a sixth order secular Hamiltonian developed with the Ellis \& Murray expansion is necessary to describe the secular dynamical behavior of HD 12661. 


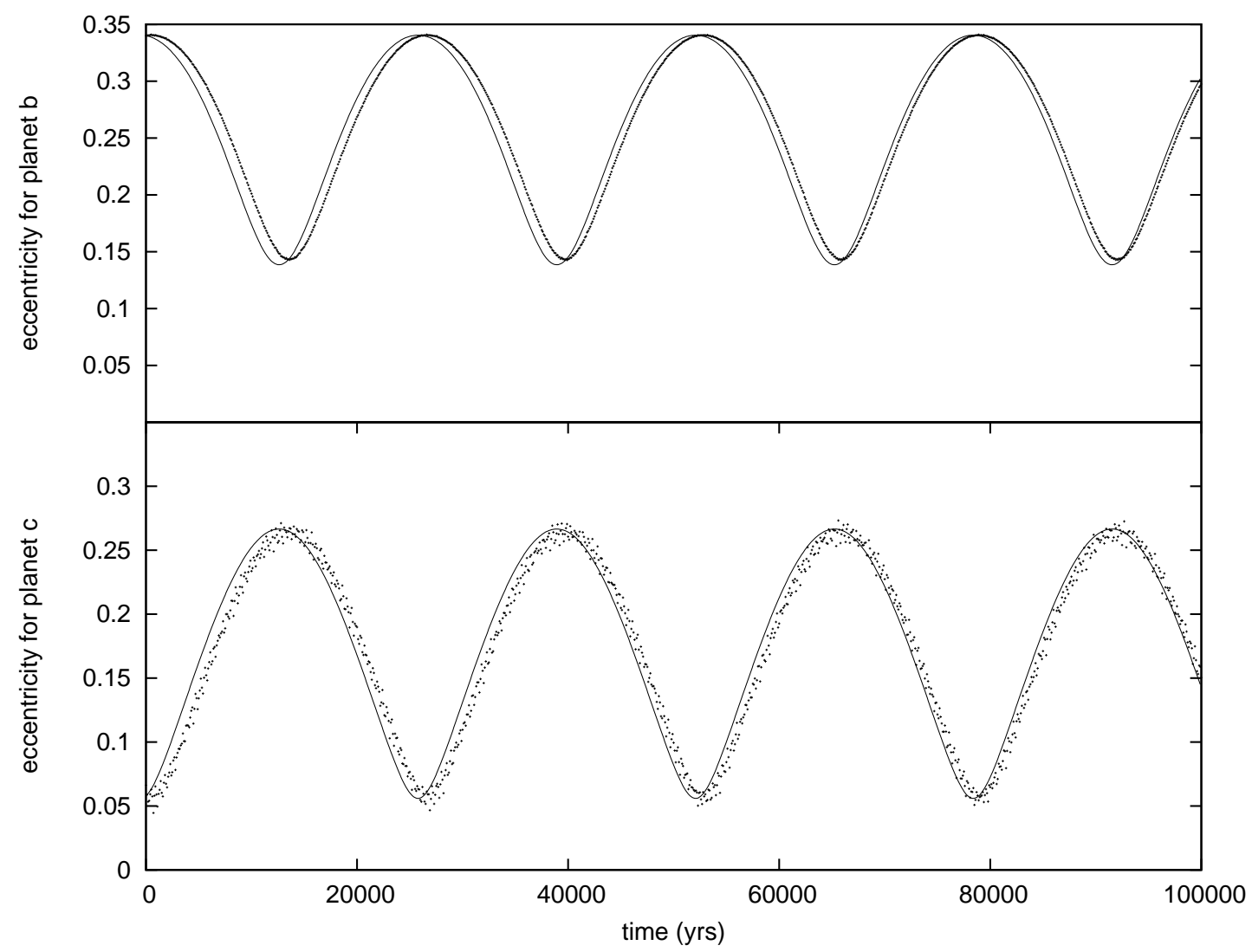

Fig. 6. - Time evolution of the eccentricities. Numerical integration (points) compared with model (lines) including up to eight order terms in eccentricities.

Figure 6 shows the time evolution (for an eight order expansion) of the eccentricities compared with the numerical integration of the full equations. Figure 7 shows for the same expansion the evolution in the plane $(k, h)$ compared also with the numerical integration of the full equations. We note that the angle $\Delta \varpi=\varpi_{1}-\varpi_{2}$ presents a libration close around $\pi$, so this shows and confirm the anti-alignment in the lines of periapses for this system.

There is an excellent agreement between the model and the numerical integration regarding the periods and the amplitudes of the oscillation of the elements. We have reproduced the numerical results through of an analytical model considering only a secular evolution of the system.

We have explored the quality of the model in the space $\left(a_{2}, e_{2}\right)$ inside the error region for initial conditions near the nominal solution and leaving unchanged the other orbital elements 


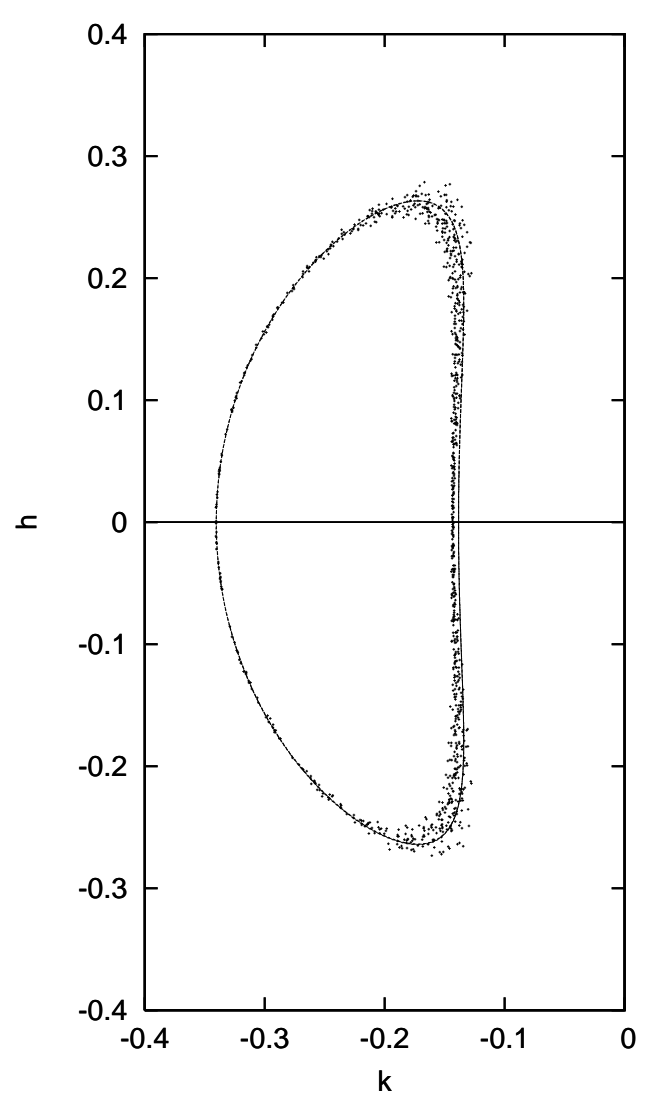

Fig. 7.- Anti-alignment of pericenters. Comparison between numerical integration (points) and model (lines) in the plane $\left(e_{1} \cos \Delta \varpi, e_{1} \sin \Delta \varpi\right)$

of the system. The results were similar to the one presented here being the differences between secular model an numerical integration of the full equations of motion of the order of $1 \%$ reaching the convergence at sixth or eight order in eccentricities.

\section{Discussion and Conclusions}

The dynamical evolution of planetary systems can be studied by means of the Lagrange's planetary equations, but if we are interested in using a Hamiltonian formalism it is necessary to work with the adequate canonical set of variables. Two sets have been proposed in the literature: Jacobi coordinates and the canonical astrocentric variables. When studying only the secular evolution, the problem is simplified considerably because of the drop of the indirect terms of the disturbing function.

The use of disturbing functions truncated to low order in eccentricities are not appro- 
priated for a correct analytical description of planetary systems with moderate eccentricities like HD 12661. For this system in particular it is necessary at least a sixth order expansion in eccentricities.

An obvious requirement is the convergence of the expansion of the disturbing function which must be previously tested. For orbits that intersects, the Ellis \& Murray expansion must be substituted for example by the one given by Beaugé \& Michtchenko (2003).

Assuming the present orbital data, with a sixth or eight order expansion of the disturbing function the secular evolution of HD 12661 is very well reproduced and it is not necessary to invoke resonant effects although it is very close to the 2:13 and 3:19 resonances. These very high order resonances cannot affect the global dynamics of the system because these resonant terms are vanishingly small but their signatures could be detected by numerical analysis methods like MEGNO (Cincotta \& Simó 2000).

The calculation and procedures described in this paper were also applied using the orbital elements used by Lee \& Peale (2003), where $a_{2}$ was taken 2.56 AU. In that paper the authors using the octopole model obtained an oscillation period of 21000 yrs contrasting with the period of 12000 yrs given by the numerical integration of the full equations. Using our approach with successive higher order expansions we obtained different oscillation periods converging to a value. For a second order expansion we obtained an oscillation period of 22200 yrs similar as these authors. Using a four order expansion we obtained a period of $15600 \mathrm{yrs}$ and with a sixth order expansion we get 14500 yrs. No notorious improvements were obtained using higher order terms. The same results were confirmed by Cristian Beaugé (2004, private communication) using his expansion. Then we obtained a better secular description than the octopole model but as these authors we also concluded that for those orbital elements, and in particular for that value of $\alpha$, there were a contribution from some resonant terms, maybe 2:11 and or 1:6 (Fig. 1). However that contribution is notoriously smaller (2500 yrs) than the one deduced from the octopole model (9000 yrs).

Theoretical analysis based on expansions truncated at small order in the eccentricities (Zhou \& Sun 2003, Lee \& Peale 2003, Nagasawa et al. 2003) can give us a qualitatively good description but fails in predicting the correct time evolution. The high eccentricities of some planetary systems requires the use of higher order classical expansions or the use of expansions suited for high eccentricities and even crossing orbits (Beaugé \& Michtchenko 2003).

Further observations and reduction methods most probably will change the nominal values for the orbital elements of this system. Then we can not pretend to have found the 
dynamical mechanism that drives the real system. But at least we have demonstrated that to have an adequate analytical model we must take into account at least up to the sixth order terms of the expansion of disturbing function. We can not accept either that the present value for $\alpha$ is the definitive one. But taking into account the errors provided by the last orbital determinations we can have an idea of the most important mean motion resonances that can affect this system (Fig. 1). Having this present, we showed that high order resonances near the system like 2:13 or 3:19 cannot affect significantly the dynamics of the system because of the very negligible magnitude of those resonant terms. This is consistent with the results of Goździewski \& Maciejewski (2003) where there is no evident sign of these resonances in their numerical analysis. The resonance 1:6 instead (Figs. 4 and 5) or less probably the 1:7, could have appreciable signatures in the dynamical evolution for the appropriate values of $\alpha$ (which is not the nominal case) specially for high enough eccentricities since the resonant terms are proportional to $e^{q}$ being $q$ the order of the resonance.

We are grateful to Debra Fischer for providing us the last orbital determinations and to Cristian Beaugé for helping us in checking our results with his proper programs and for fruitful discussions. We also acknowledge to an anonymous referee that pointed out some important observations to the original version. 


\section{REFERENCES}

Beaugé, C., \& Michtchenko, T. A. 2003, MNRAS, 341, 760

Brouwer, D. \& Clemence, G. M. 1961. Methods of Celestial Mechanics. Academic Press, New York.

Chambers, J. E. 1999, MNRAS, 304, 793

Cincotta, P. M. \& Simó, C. 2000, A\&AS, 147, 205

Ellis, K. M., \& Murray, C. D. 2000, Icarus, 147, 129

Ferraz-Mello, S., Michtchtenko, T. A., \& Beaugé, C. 2004, to be published in "Chaotic Worlds: From Order to Disorder in Gravitational N-Body Systems" (B. A., Steves, ed.), Kluwer Acad. Publ.

Fischer, D. A., Marcy G. W., Butler, R. P., Vogt, S. S., Henry, G. W., Pourbaix, D., Walp, B., Misch, A. A., \& Wright, J. 2003, ApJ, 586, 1394

Gallardo, T., \& Ferraz-Mello, S. 1997, AJ, 113(2), 863

Goździewski, 2003, A\&A, 398, 1151

Goździewski, K., \& Maciejewski, A. J. 2003, ApJ, 586, L153

Hadjidemetriou, J. 2002, Cel. Mech. \& Dynam. Astron., 83, 141

Ji, J. et al. 2003, ApJ, 591, L57

Laskar, J., \& Robutel, P. 1995, Cel. Mech. \& Dynam. Astron., 62, 193

Lee, M. H., Peale, S. J. 2003, ApJ, 592, 1201

Lissauer, J.J. \& Rivera, E . J. 2001, ApJ, 554, 1141.

Michtchenko, T. A., \& Ferrz-Mello, S. 2001, Icarus, 149, 357

Michtchenko, T. A., \& Malhotra, R. 2004, Icarus, 168, 237

Morbidelli, A. 2002, Modern Celestial Mechanics. Taylor \& Francis.

Murray, C. D., \& Dermott, S.F. 1999, Solar System Dynamics. Cambridge University Press.

Nagasawa, M., Lin, D. N. C., \& Ida, S. 2003, ApJ, 586, 1374 
Papaloizou, J. C. B. 2003, Cel. Mech. \& Dynam. Astron. , 87, 53

Zhou, J. L., \& Sun, Y. S. 2003, ApJ, 598, 1290 